\title{
Symmetric hyperbolic form of systems of second-order evolution equations subject to constraints
}

\author{
Carsten Gundlach $*$ \\ School of Mathematics, University of Southampton, Southampton SO17 1BJ, UK \\ José M. Martín-Garcídi \\ Instituto de Matemáticas y Física Fundamental, Centro de Física Miguel A. Catalán, \\ C.S.I.C., Serrano 113 bis, 28006 Madrid, Spain
}

(Dated: 18 February 2004)

\begin{abstract}
Motivated by the initial-boundary value problem for the Einstein equations, we propose a definition of symmetric hyperbolicity for systems of evolution equations that are first order in time but second order in space. This can be used to impose constraint-preserving boundary conditions. The general methods are illustrated in detail in the toy model of electromagnetism.
\end{abstract}

\section{INTRODUCTION}

In attempting to solve the Einstein equations numerically as an initial or initial-boundary value problem, it is important to start with a well-posed continuum problem. For systems of first-order equations, there are two useful criteria for well-posedness: Strong hyperbolicity is a necessary and sufficient criterion for the initial value problem (without boundaries or with periodic boundaries) to be well-posed. Roughly speaking, it means that the system possesses a complete set of characteristic variables, which propagate with definite speeds. Symmetric hyperbolicity implies strong hyperbolicity, and can be used to formulate a well-posed initial-boundary value problem. Roughly speaking, it means that the principal part of the system possesses an (unphysical) energy whose growth due to boundary terms can be expressed as "incoming mode energy minus outgoing mode energy".

Much less is known about how to make systems of second-order equations well-posed. Here we concentrate on systems which have been brought into first order in time but remain second order in space. The simplest example of such a system is the wave equation, written as

$$
\begin{aligned}
\partial_{t} \phi & =\Pi, \\
\partial_{t} \Pi & =\partial_{i} \partial^{i} \phi .
\end{aligned}
$$

The Arnowitt-Deser-Misner (ADM) formulation of the Einstein equations has a similar form, with the 3-metric corresponding to $\phi$ and the extrinsic curvature to $\Pi$.

In this paper we discuss three methods for investigating and proving well-posedness for such systems: we review two known methods in order to put our work into context, and then propose one which we believe to be new and advantageous.

The first old method, (differential) reduction to first order, introduces the spatial derivatives as auxiliary vari-

*C.Gundlach@maths.soton.ac.uk

†jmm@imaff.cfmac.csic.es ables. For the wave equation above, these could be called $d_{i} \equiv \partial_{i} \phi$. One then has a larger but first-order system, which one can hope to make strongly or symmetric hyperbolic. A drawback of this method is that its solution space is larger than that of the original system: it is equivalent to the original system only if the three auxiliary constraints $C_{i} \equiv d_{i}-\partial_{i} \phi$ vanish. Therefore proving strong or symmetric hyperbolicity for the first-order reduction does not prove well-posedness for the original second-order system except in simple cases where the auxiliary constraints evolve trivially. (These include the wave equation and electromagnetism, but not the full Einstein equations.)

A second method called pseudo-differential reduction to first order Fourier-transforms the PDE system in space. If it is linear with constant coefficients, one effectively obtains a system of decoupled ODEs, one for each wave number $\omega_{i}$. One can then show a version of strong hyperbolicity for this system. The key point is that the pseudo-differential reduction does not introduce any auxiliary variables. Proving strong hyperbolicity therefore also proves well-posedness of the initial value problem for the original second-order system 1]. This method has been applied to a formulation of the Einstein equations in 2]. A drawback is that as the Fourier transform is nonlocal, one cannot find a locally conserved energy in this way, and so cannot address the initial-boundary value problem.

In order to avoid the drawbacks of these two approaches, we propose a fairly obvious generalisation of the concept of symmetric hyperbolicity to systems that are second order in space and first order in time. In the example of the wave equation, we define characteristic variables in a given direction $n_{i}$ as $\Pi \pm n^{i} \partial_{i} \phi$, and an energy density $\Pi^{2}+\left(\partial_{i} \phi\right)^{2}$. The energy and its associated flux can be written in terms of characteristic variables, and so we can impose maximally dissipative boundary conditions similar to those for a first-order symmetric hyperbolic system.

The paper is organised as follows: In Sec. II we review strong and symmetric hyperbolicity, and the initialboundary value problem, for first-order systems. In 
Sec. III we review pseudo-differential reduction to first order. In Sec. IV we present our concept of symmetric hyperbolicity for a system that is second order in space and first order in time. In Sec. $\nabla]$ we review the initialboundary value problem for systems with differential constraints, such as the Maxwell or Einstein equations. This discussion applies both to first and to second order symmetric hyperbolic systems.

Finally, in Sec.VI we compare the three different methods by applying them to the Maxwell equations: reduction to first order with auxiliary variables, pseudodifferential reduction to first order, and our method of finding characteristic variables and an energy directly for the second-order system. We formulate constraintpreserving boundary conditions, show that the initialboundary value problem for the second-order Maxwell equations can be made well-posed for "Neumann" and "Dirichlet" boundary conditions, and conjecture that it is well-posed for a one-parameter family of boundary conditions which interpolates between those two via "Sommerfeld" boundary conditions.

\section{FIRST-ORDER SYSTEMS}

In this section we summarise some general results on the well-posedness of systems of first-order evolution equations that are proved for example in 3]. We begin by considering linear systems with constant coefficients, and at the end of the section appeal to standard methods for generalising these results to quasilinear systems. (For other reviews, see [4, 5].)

\section{A. Strong hyperbolicity}

Consider the system of first-order linear evolution equations with constant coefficients

$$
\partial_{t} u=P^{i} \partial_{i} u+Q u
$$

Here $u$ is a vector of variables, $P^{i}$ and $Q$ are square matrices, and $i$ ranges over the spatial directions. The initialvalue problem for such a system is called well-posed if a solution exists, is unique and depends continuously on the initial data $u(x, 0)$. This is equivalent to there being an estimate

$$
\|u(\cdot, t)\| \leq K e^{\alpha t}\|u(\cdot, 0)\|
$$

with respect to an $L_{2}$ norm, where the constants $K$ and $\alpha$ do not depend on the initial data. To simplify the presentation, in the following we shall assume that $Q=0$. Leaving $Q$ in the calculation would show that it influences the value of $\alpha$, but not the existence of the estimate itself.

The main result is that the existence of an estimate (4) is equivalent to the system being strongly hyperbolic: for any unit covector $n_{i}$, the matrix $P_{n}=n_{i} P^{i}$ has only real eigenvalues and a complete set of real eigenvectors.
The idea of the proof of this theorem is to go to Fourier space. Let $\hat{u}(\omega, t)$ be the Fourier transform of $u(x, t)$ :

$$
u(x, t) \equiv \int_{R^{3}} \hat{u}(\omega, t) e^{i \omega_{i} x^{i}} \frac{d^{3} \omega}{(2 \pi)^{3 / 2}} .
$$

Parseval's theorem states that $\|u\|_{x}=\|\hat{u}\|_{\omega}$ and so the estimate (4) is equivalent to

$$
\|\hat{u}(\cdot, t)\| \leq K e^{\alpha t}\|\hat{u}(\cdot, 0)\|
$$

The system in $x$ and $t$ becomes an ODE system for each value of $\omega_{i}$ :

$$
\partial_{t} \hat{u}=i|\omega| P_{n} \hat{u}
$$

where now $n_{i}=\omega_{i} /|\omega|$. Now, if $P_{n}$ has complex eigenvalues, then $\alpha$ depends on the largest $|\omega|$ present in the initial data, and hence on the initial data. If $P_{n}$ lacks a complete set of eigenvectors, the normal form of $P_{n}$ contains at least one Jordan block of size $k>1$. One can show that then $|\hat{u}(\omega, t)|$ is bounded only by $|\omega t|^{k-1}$, so that now $K$ depends on the largest value of $|\omega|$ present in the initial data.

On the other hand, if $P_{n}$ is diagonalisable with real eigenvalues, let $\Lambda_{n}$ be the diagonal matrix of eigenvalues, and $T_{n}$ be a matrix of the corresponding right (column) eigenvectors of $P_{n}$,

$$
P_{n} T_{n}=T_{n} \Lambda_{n}
$$

(Note that $T_{n}$ is not unique.) The Fourier characteristic variables

$$
\hat{U}(\omega, t) \equiv T_{n}^{-1} \hat{u}(\omega, t)
$$

obey

$$
\partial_{t} \hat{U}=i|\omega| \Lambda_{n} \hat{U}
$$

This gives

$$
|\hat{U}(\omega, t)|=|\hat{U}(\omega, 0)|
$$

and so

$$
|\hat{u}(\omega, t)| \leq\left|T_{n}\right|\left|T_{n}^{-1}\right||\hat{u}(\omega, 0)| .
$$

If $T_{n}$ and $T_{n}^{-1}$ are continuous in $n_{i}$, we obtain (6) and hence (4), with $\alpha=0$ and $K=\sup \left(\left|T_{n}\right|\left|T_{n}^{-1}\right|\right)$.

We now go back to real space, and define the vector $U$ of characteristic variables with respect to an arbitrary fixed direction $n_{i}$ by

$$
U \equiv T_{n}^{-1} u
$$

The characteristic variables always depend on the direction $n_{i}$, but following standard notation we do not indicate this explicitly. We define the derivative in the direction $n_{i}$ as

$$
\partial_{n} \equiv n^{i} \partial_{i}
$$


and the projector $q^{i}{ }_{j}$ into the space normal to $n_{i}$ by

$$
q_{j}^{i} \equiv \delta_{j}^{i}-n^{i} n_{j}
$$

(Note that we need a spatial metric to define $n^{i}$.) We also define the shorthand index notation $A$ for a tensor index $i$ that has been projected with $q^{i}{ }_{j}$, for example in

$$
P^{i} q_{i}{ }^{j} \partial_{j} \equiv P^{A} \partial_{A}
$$

With this notation we can write

$$
P^{i} \partial_{i}=P_{n} \partial_{n}+P^{A} \partial_{A}
$$

The characteristic variables obey

$$
\partial_{t} U=\Lambda_{n} \partial_{n} U+\left(T_{n}^{-1} P^{A} T_{n}\right) \partial_{A} U
$$

Strong hyperbolicity implies the existence of a symmetriser, which is a Hermitian matrix $H_{n}$ for every unit covector $n_{i}$ that obeys

$$
\left(H_{n} P_{n}\right)^{\dagger}=H_{n} P_{n}
$$

The general matrix $H_{n}$ with this property can be constructed as

$$
H_{n}=\left(T_{n}^{-1}\right)^{\dagger} B_{n} T_{n}^{-1}
$$

where $B_{n}$ is Hermitian, positive definite, and commutes with $\Lambda_{n}$. The general matrix $B_{n}$ with this last property is block-diagonal, with each block corresponding to one (possibly multiple) eigenvalue in $\Lambda_{n}$. For given $T_{n}, H_{n}$ is parameterised by $B_{n}$. Conversely, it can be parameterised by $T_{n}$ with $B_{n}=I$. If the field equations do not single out a preferred direction $n_{i}$, then $\Lambda_{n}$ do not depend on $n_{i}$, and it is natural to choose $T_{n}$ so that $B_{n}$ is also independent of $n_{i}$. We then write $B$ and $\Lambda$ instead of $B_{n}$ and $\Lambda_{n}$.

\section{B. Symmetric hyperbolicity}

We shall now see how well-posedness can be maintained in the presence of boundaries in space. Without loss of generality we assume that these boundaries are fixed (at constant values of the coordinates $x^{i}$.) If a symmetriser $H_{n}=H$ exists that is independent of $n_{i}$, the system is called symmetric hyperbolic. In this case the system admits a positive definite energy

$$
E=\int_{\Omega} \epsilon d V, \quad \epsilon=u^{\dagger} H u .
$$

This means that $E$ is conserved up to boundary terms: Assuming still that $H$ and $P^{i}$ are constant, and with $H P^{i}=\left(H P^{i}\right)^{\dagger}$, we have

$$
\partial_{t} \epsilon=\partial_{i}\left(u^{\dagger} H P^{i} u\right)
$$

and so by Gauss' law

$$
\frac{d E}{d t}=\int_{\partial \Omega} F^{i} d S_{i}, \quad F^{i}=u^{\dagger} H P^{i} u,
$$

where $d S_{i}$ is the surface element on the boundary $\partial \Omega$. This can be written as

$$
\frac{d E}{d t}=\int_{\partial \Omega} F^{n} d S, \quad F^{n}=U^{\dagger} B \Lambda U,
$$

where $n_{i}$ is the normal to $\partial \Omega$.

The boundary flux can be written schematically as " $U_{+}^{2}-U_{-}^{2} "$, or more precisely as

$$
F^{n}=U_{+}^{\dagger} B_{+} \Lambda_{+} U_{+}+U_{-}^{\dagger} B_{-} \Lambda_{-} U_{-}
$$

where $U_{+}$are modes that come in across the boundary and $U_{-}$are outgoing, and where $B_{ \pm}$and $\Lambda_{ \pm}$are the blocks corresponding to $U_{ \pm}$. Therefore $B_{+} \Lambda_{+}$is positive definite and $B_{-} \Lambda_{-}$is negative definite (the meaning of the minus sign in " $U_{+}^{2}-U_{-}^{2}$ "). There may also be modes $U_{0}$ whose propagation speed across the boundary is zero. In this case the boundary is called characteristic. These modes do not contribute to $F^{n}$. It is clear that boundary conditions must be imposed on the incoming variables $U_{+}$, and that no boundary conditions can be imposed on the variables $U_{-}$and $U_{0}$. In the following we assume that the number of variables that propagate along the boundary is constant.

Estimates of the type (4) can be obtained from an energy $E$ whose growth is suitably bounded. One can achieve this by imposing a maximally dissipative boundary condition

$$
U_{+}=L U_{-}+f
$$

where $f$ is given and the matrix $L$ is sufficiently small. For $f=0$ this condition means that more energy goes out than comes in at every point on the boundary (not just integrated over the boundary). For $f \neq 0$ given, the growth of $E$ can be bounded from $f$. The required smallness condition on the matrix $L$ is that the matrix

$$
L^{\dagger} B_{+} \Lambda_{+} L+B_{-} \Lambda_{-}
$$

is negative definite. For maximally dissipative boundary conditions to result in a well-posed initial-boundary value problem, the system has to be symmetric hyperbolic. There are examples where imposing them on a strongly but not symmetric hyperbolic system leads to an ill-posed problem [6].

\section{Variable coefficient and quasilinear systems}

The results on linear systems with constant coefficients can be generalised to linear systems with variable coefficients 3]. It is sufficient that the system is strongly or symmetric hyperbolic at every point in space and time 
(frozen coefficients approximation), and bounds can be obtained that are uniform over all space, all directions $n_{i}$, and a finite time interval. In obtaining an energy for the system with variable coefficients, the time and space derivatives of the non-constant coefficients give rise to non-principal terms, but these do not affect wellposedness.

The results for linear problems with variable coefficients can be extended to nonlinear problems. In a first step, linearising a non-linear problem around a background solution gives rise to a linear problem with variable coefficients. Clearly a necessary condition for the non-linear problem to be well-posed is that the linearised problem is well-posed. Furthermore, if the linearised problem is well-posed for all backgrounds, then it may be possible to prove well-posedness for the nonlinear problem [3].

Physically, the frozen coefficients, principal part only approximation means that we focus on possible instabilities in small high-frequency perturbations. A problem is ill-posed in practice because it allows perturbation modes whose growth rate is not bounded - the higher the frequency in space, the faster the growth in time. In numerical simulations such modes show up as instabilities at the grid frequency which grow faster at higher resolution, thus destroying convergence at sufficiently late times and high resolutions. Well-posedness does not rule out instabilities (in the loose sense of rapidly growing perturbations, possibly violating the constraints) which are generated by non-principal terms and whose growth is bounded independently of spatial frequency.

\section{PSEUDO-DIFFERENTIAL REDUCTION TO FIRST ORDER}

Pseudo-differential reduction to first order has recently been suggested as a technique for proving that certain second-order evolution systems are strongly hyperbolic [1]. We review it here only to see how it relates to the two other ways of dealing with a second-order system: reduction to a first-order system by introducing auxiliary variables, and the second-order energy method we propose in this paper.

A system of first order pseudo-differential equations is one whose Fourier transform in space is of the form (7) but where $P_{n}$ is not given by the linear expression $P_{n}=n_{i} P^{i}$ for a constant $P^{i}$. The definition of strong hyperbolicity of a system of evolution equations generalises to the pseudo-differential case, and strong hyperbolicity is again equivalent to well-posedness (in the absence of boundaries), because the proof using the ODE system (7) refers only to the matrix $P_{n}$ but not directly to the vector of matrices $P^{i}$.

First-order pseudo-differential systems can be derived from a class of second-order differential systems. The simplest example is the wave equation, in the form (12).
The Fourier transform of this system is

$$
\begin{aligned}
\partial_{t} \hat{\phi} & =\hat{\Pi} \\
\partial_{t} \hat{\Pi} & =-|\omega|^{2} \hat{\phi} .
\end{aligned}
$$

Replacing $\hat{\phi}$ by $\hat{\varphi} \equiv i|\omega| \hat{\phi}[2]$, we obtain the first-order pseudo-differential system

$$
\begin{aligned}
& \partial_{t} \hat{\varphi}=i|\omega| \hat{\Pi}, \\
& \partial_{t} \hat{\Pi}=i|\omega| \hat{\varphi} .
\end{aligned}
$$

Note that this is not the Fourier transform of a differential system as $P_{n}$ is independent of $n_{i}$. This system is strongly hyperbolic, with Fourier characteristic variables

$$
\hat{U}_{ \pm}=\hat{\Pi} \pm \hat{\varphi}
$$

which obey

$$
\partial_{t} \hat{U}_{ \pm}= \pm i|\omega| \hat{U}_{ \pm}
$$

\section{SECOND ORDER IN SPACE, FIRST ORDER IN TIME}

We shall now show how the two key ideas for first-order systems - strong and symmetric hyperbolicity - can be adapted to a system that is first order in time but second order in space.

\section{A. Strong hyperbolicity}

In analogy with (18) we define a linear combination $U$ of $\tilde{u} \equiv\left(u, \partial_{i} u\right)$ to be a characteristic variable of the second order system with speed $\lambda$ in the direction $n_{i}$ if it obeys

$$
\partial_{t} U=\lambda \partial_{n} U+\text { transversal derivatives. }
$$

We illustrate this again for the scalar field model. The second order characteristic variables are

$$
\begin{aligned}
U_{ \pm} & \equiv \Pi \pm \partial_{n} \phi \\
U_{A} & \equiv \partial_{A} \phi
\end{aligned}
$$

and obey

$$
\begin{aligned}
& \partial_{t} U_{ \pm}= \pm \partial_{n} U_{ \pm}+\partial^{A} U_{A} \\
& \partial_{t} U_{A}=\partial_{A} \frac{1}{2}\left(U_{+}+U_{-}\right)
\end{aligned}
$$

Note that one can obtain the non-zero speed characteristic variables (35) of the second order PDE system from those of the pseudo-differential system, (32), because both are eigenvectors of essentially the same matrix $P_{n}$. In the second-order system we neglect the transverse derivatives to find $P_{n}$, while in the pseudo-differential system $n_{i}$ is defined as $\omega_{i} /|\omega|$, and so the transversal derivatives vanish by definition. 
The definition (34) of characteristic variables for a second-order in space system has two consequences which are not apparent in the simple scalar field example. The first is that a transversal derivative $\partial_{A} u$ is by definition a zero-speed characteristic variable because we can interchange derivatives:

$$
\partial_{t}\left(\partial_{A} u\right)=\partial_{A}\left(\partial_{t} u\right)
$$

The second is that all characteristic variables of a secondorder system are unique only up to addition of transversal derivatives. This non-uniqueness will later be used up to write $\epsilon$ and $F^{n}$ in terms of characteristic variables.

\section{B. Symmetric hyperbolicity}

We would like to generalise the energy (21) to a system that is second order in space. As in the first order system, we require an energy density and flux that obey the conservation law

$$
\partial_{t} \epsilon=\partial_{i} F^{i}
$$

and can be expressed in characteristic variables.

We demonstrate the second-order energy and maximally dissipative boundary conditions for the scalar field. The scalar field admits a positive definite conserved energy that is unique up to an overall scale, with energy density

$$
\epsilon=\Pi^{2}+\left(\partial_{i} \phi\right)^{2}
$$

and flux

$$
F^{i}=2 \Pi \partial^{i} \phi
$$

The energy can be written as

$$
\epsilon=\frac{1}{2}\left(U_{+}^{2}+U_{-}^{2}\right)+U_{A} U^{A}
$$

and the flux in the direction $n_{i}$ normal to the boundary can be written as

$$
F^{n}=\frac{1}{2}\left(U_{+}^{2}-U_{-}^{2}\right) .
$$

This means that

$$
U_{+}=\kappa U_{-}+f
$$

with $|\kappa| \leq 1$ is a maximally dissipative boundary condition for the system (12). As the characteristic variables of the second-order system contain derivatives, this is not an algebraic but a first-order differential boundary condition, namely

$$
(1-\kappa) \Pi+(1+\kappa) \partial_{n} \phi=f
$$

We see that $\kappa=-1$ corresponds to an (inhomogeneous) Dirichlet boundary condition on $\Pi$ and hence on $\phi, \kappa=1$ to Neumann, and $\kappa=0$ to specifying the ingoing radiation (Sommerfeld).

The conserved energy with $\epsilon$ given by (41) can be used to bound the $L_{2}$ norms of $\Pi$ and $\partial_{i} \phi$, but not $\|\phi\|$ itself. To obtain a bound on $\|\phi\|$ as well, we can use the energy given by

$$
\tilde{\epsilon}=\Pi^{2}+\left(\partial_{i} \phi\right)^{2}+\alpha^{2} \phi^{2}
$$

with $\alpha>0$. Clearly this gives us

$$
\|\Pi\| \leq \tilde{E}^{1 / 2}, \quad\left\|\partial_{i} \phi\right\| \leq \tilde{E}^{1 / 2}, \quad\|\phi\| \leq \alpha^{-1} \tilde{E}^{1 / 2},
$$

but with the disadvantage that $\tilde{E}$ is not strictly conserved. Rather

$$
\frac{d \tilde{E}}{d t}=\int_{\partial \Omega} 2 \Pi \partial^{i} \phi d S_{i}+2 \alpha^{2} \int_{\Omega} \Pi \phi d V \leq 2 \alpha \tilde{E}
$$

where we have assumed maximally dissipative boundary conditions to make the boundary term non-positive. This is an inefficient estimate, but $\alpha$ can be made arbitrarily small. Non-principal terms in the equations can be dealt with in a similar manner: they give rise to volume terms in $d E / d t$ that can be estimated as a multiple of $E$ itself. (Note that the issues of bounding $\|\phi\|$ and of dealing with non-principal terms are dealt with identically in secondorder and first-order systems.)

\section{CONSTRAINT-PRESERVING BOUNDARY CONDITIONS}

Here we summarise a general method of dealing with a system of evolution equations that are subject to differential constraints (7] and references there). As the constraints are by assumption compatible with the evolution equations, they form a closed evolution system. We now assume that this constraint system and the main system are symmetric hyperbolic. The following discussion applies then equally to first-order and second-order systems.

Rather than defining a notation for the general case, we consider the case where the non-zero speed characteristic variables come in pairs with speeds $\pm \lambda$, with $\lambda>0$, plus a number of zero-speed variables. (This is necessarily true if there is no preferred direction in space.) Focus on a pair of characteristic constraint variables $C_{ \pm}$which obey

$$
\partial_{t} C_{ \pm}= \pm \lambda \partial_{n} C_{ \pm}+\ldots
$$

where ... stands for transversal derivatives and any nonprincipal terms. As the constraints are compatible with the evolution equations, there exists a pair of characteristic variables of the main system $U_{ \pm}$with the same speeds, that is

$$
\partial_{t} U_{ \pm}= \pm \lambda \partial_{n} U_{ \pm}+\ldots
$$


such that

$$
C_{ \pm}=\partial_{n} U_{ \pm}+\ldots
$$

The constraint energy is initially zero and we want it to remain zero. One way of enforcing that is to formally impose homogeneous maximally dissipative boundary conditions on the constraint system, that is

$$
C_{+}=\kappa C_{-}
$$

for $|\kappa| \leq 1$. If we now define

$$
X \equiv U_{+}-\kappa U_{-},
$$

the boundary condition on the constraint system (53) is equivalent to the boundary condition

$$
\partial_{n} X=\ldots
$$

on the main system, taking into account the relation (52). We now use the evolution equations for $U_{ \pm}$to replace $\partial_{n}$ with $\partial_{t}$, and find an evolution equation for $X$ which contains only transversal derivatives (derivatives parallel to the boundary):

$$
\partial_{t} X=\ldots
$$

The trick is to interpret this as an evolution equation for an auxiliary variable $X$ which is defined only on the boundary. (In general, there will be more than one pair of constraints, and a system of variables $X$.) The boundary condition

$$
U_{+}=\kappa U_{-}+X
$$

has the form of a maximally dissipative boundary condition on $U$, but it is one properly speaking only if $X$ can be treated as given. This is the case only if the boundary system (56) decouples from the bulk system.

One can show that, with maximally dissipative boundary conditions,

$$
\begin{aligned}
\frac{d}{d t}\|X\|_{\partial \Omega} & \leq K_{1}\|f\|_{\partial \Omega}+K_{2}\|u\|_{\partial \Omega}, \\
\frac{d}{d t}\|u\|_{\Omega} & \leq K_{3}\|X\|_{\partial \Omega} .
\end{aligned}
$$

If the boundary system decouples from the bulk system, $K_{2}=0$ and the growth of the boundary solution and hence of the bulk solution is bounded by $f$. But a bound on $\|u\|_{\Omega}$ does not imply one on $\|u\|_{\partial \Omega}$, and so for $K_{2} \neq 0$ well-posedness cannot be proved using these energy techniques.

\section{THE MAXWELL EQUATIONS}

\section{A. Field equations}

The Maxwell (electromagnetism, EM) equations in the presence of a charge density $\rho$ and current density $j_{i}$ can be written as the evolution equations

$$
\begin{aligned}
\partial_{t} A_{i} & =-E_{i}-\partial_{i} \psi, \\
\partial_{t} E_{i} & =-\partial_{j} \partial^{j} A_{i}+\partial_{i} \partial^{j} A_{j}-4 \pi j_{i},
\end{aligned}
$$

subject to a constraint

$$
C_{E} \equiv \partial^{i} E_{i}-4 \pi \rho=0 .
$$

Here $\psi, j_{i}$ and $\rho$ can be treated as given functions, subject to the charge conservation law $\partial_{t} \rho+\partial^{i} j_{i}=0$. All indices are moved with the flat Cartesian spatial metric $\delta_{i j}$, and staggered double indices are summed over.

The EM system would be a wave equation for $A_{i}$ were it not for the grad $\operatorname{div}$ term $\partial_{i} \partial^{j} A_{j}$. This term can be eliminated by making the offending divergence a new variable. This property makes the EM system a nice toy model for the ADM formulation of the Einstein equations [8]. We therefore introduce $\Gamma \equiv \partial^{i} A_{i}$. This gives rise to the new constraint

$$
C_{\Gamma} \equiv \Gamma-\partial^{i} A_{i}=0
$$

We obtain an evolution equation for $\Gamma$ by taking the divergence of the evolution equation for $A_{i}$, and add to it $b C_{E}$ with a free coefficient $b$. We also add $a \partial_{i} C_{\Gamma}$ to the evolution equation for $E_{i}$ with a free coefficient $a$. The resulting system is

$$
\begin{aligned}
\partial_{t} A_{i} & =-E_{i}-\partial_{i} \psi \\
\partial_{t} E_{i} & =-\partial_{j} \partial^{j} A_{i}+a \partial_{i} \Gamma+(1-a) \partial_{i} \partial^{j} A_{j}-4 \pi j_{i}, \\
\partial_{t} \Gamma & =(b-1) \partial_{i} E^{i}-b 4 \pi \rho-\partial_{i} \partial^{i} \psi .
\end{aligned}
$$

Note that for $a=0, \Gamma$ decouples and we recover the original system. For $a=1, A_{i}$ obeys a wave equation. The constraint system is

$$
\begin{aligned}
\partial_{t} C_{E} & =a \partial_{i} \partial^{i} C_{\Gamma}, \\
\partial_{t} C_{\Gamma} & =b C_{E} .
\end{aligned}
$$

In the following, we concentrate on the principal part. In the EM system, this means neglecting the terms containing $\rho, j_{i}$ and $\psi$. (For the EM system the principal part approximation happens to be exact: it corresponds to vacuum electrodynamics in the gauge $\psi=0$.)

We now use the Maxwell equations to illustrate three methods of investigating well-posedness: differential reduction to first order, pseudo-differential reduction, and making the original second-order system symmetric hyperbolic. This is only for illustration: our preferred method is the last one. We have introduced the parameters $a$ and $b$ to clarify the presentation, and because precisely analogous parameters exist for the ADM formulation of the Einstein equations. We shall see that a natural choice of these parameters would be $a=b=1$. 


\section{B. Differential reduction to first order}

\section{Field equations}

To reduce the EM main system to first order, we introduce the auxiliary variables $d_{i j} \equiv \partial_{i} A_{j}$. Because $A_{i}$ does not appear undifferentiated in the field equations, this definition itself does not appear as a new constraint, but its integrability condition does:

$$
C_{i j k} \equiv \partial_{i} d_{j k}-\partial_{j} d_{i k}=0 .
$$

The only term in the second-order system that does not translate unambiguously into the new variables is $\partial_{i} \partial^{j} A_{j}$, which can be written either as $\partial_{i} d_{j}{ }^{j}$ or $\partial^{j} d_{i j}$. We therefore parameterise this choice with a parameter $c$. From the point of view of the new system, this can be described as adding the constraint $-c C_{i j}{ }^{j}$ to the evolution equation for $E_{i}$ with a free coefficient $c$. The resulting system is (now neglecting non-principal terms as discussed above)

$$
\begin{aligned}
\partial_{t} d_{i j} & =-\partial_{i} E_{j}, \\
\partial_{t} E_{i} & =-\partial^{j} d_{j i}+a \partial_{i} \Gamma+(1-a-c) \partial_{i} d_{j}{ }^{j}+c \partial^{j} d_{i j} \\
\partial_{t} \Gamma & =(b-1) \partial_{i} E^{i}
\end{aligned}
$$

To reduce the constraint system to first order, we introduce the new variables $C_{i} \equiv \partial_{i} C_{\Gamma}$, where now $C_{\Gamma}=\Gamma-d_{j}{ }^{j}$. As $C_{\Gamma}$ does not appear in undifferentiated form, this definition itself does not appear as a constraint, but it gives rise to the integrability condition

$$
C_{i j} \equiv \partial_{i} C_{j}-\partial_{j} C_{i}=0 .
$$

The first-order constraint system is

$$
\begin{aligned}
\partial_{t} C_{E} & =a \partial^{i} C_{i}+(1-c) \partial^{i} C_{i j}{ }^{j} \\
\partial_{t} C_{i} & =b \partial_{i} C_{E} \\
\partial_{t} C_{i j k} & =0 \\
\partial_{t} C_{i j} & =0
\end{aligned}
$$

\section{Strong hyperbolicity}

We write the first-order system as

$$
\partial_{t} u=P_{n} \partial_{n} u+P^{A} \partial_{n} A u .
$$

To diagonalise $P_{n}$, we split $d_{i j}$ into the tensor components $d_{n n}, d_{q q}, d_{A n}, d_{n A}$ and $d_{A B}$ with respect to a fixed direction $n_{i}$, where the index $n$ means a contraction with $n^{i}$, the index pair $q q$ means a contraction with $q^{i j}$, and indices $A, B$, etc are the same as $i, j$ etc, but denote a tensor that is transversal and tracefree on any index pair. (Because it is transversal, it is tracefree with respect to both $\delta_{i j}$ and $q_{i j}$.) Note that $d_{i}{ }^{i}=d_{n n}+d_{q q}$. A similar tensor decomposition is used for the other variables and throughout this paper. We shall loosely refer to these objects as scalars if they have no free (transversal) index, vectors if they have one, and tensors if they have more than one.

By applying the same tensor decomposition to the evolution equations, we find $P_{n}$ in this basis: it is block-diagonal with blocks corresponding to the scalars $\left(d_{n n}, d_{q q}, E_{n}, \Gamma\right)$, transverse vectors $\left(d_{n A}, d_{A n}, E_{A}\right)$ and transverse tracefree tensors $\left(d_{A B}\right)$. The free transversal indices behave trivially, so that the blocks we still have to diagonalise are quite small. The scalar block of $P_{n}$ is given by

$$
\begin{aligned}
P_{n} d_{n n} & =-E_{n} \\
P_{n} d_{q q} & =0 \\
P_{n} E_{n} & =a\left(\Gamma-d_{n n}-d_{q q}\right)+(1-c) d_{q q}, \\
P_{n} \Gamma & =(b-1) E_{n},
\end{aligned}
$$

the vector block by

$$
\begin{aligned}
P_{n} d_{n A} & =-E_{A}, \\
P_{n} d_{A n} & =0, \\
P_{n} E_{A} & =-d_{n A}+c d_{A n},
\end{aligned}
$$

and the tensor block by $P_{n} d_{A B}=0$. The characteristic variables obey $P_{n} U=U \Lambda$ and comprise the four scalars

$$
\begin{aligned}
& d_{q q}, \\
U_{0} \equiv & \Gamma+(b-1) d_{n n}, \\
U_{ \pm} \equiv & a\left(\Gamma-d_{n n}-d_{q q}\right)+(1-c) d_{q q} \pm \sqrt{a b} E_{n},
\end{aligned}
$$

with speeds $(0,0, \pm \sqrt{a b})$, six vector components

$$
\begin{gathered}
d_{A n}, \\
U_{ \pm A} \equiv d_{n A}-c d_{A n} \mp E_{A},
\end{gathered}
$$

with speeds $(0, \pm 1)$, and three tensor components $d_{A B}$ with zero speed. The characteristic variables for the constraints are

$$
\begin{gathered}
C_{A}, \quad C_{i j}, \quad C_{i j k}, \\
C_{ \pm} \equiv a C_{n}+(1-c) C_{n i}{ }^{i} \pm \sqrt{a b} C_{E},
\end{gathered}
$$

with speeds $(0, \pm \sqrt{a b})$. (We have not bothered to decompose $C_{i j}$ and $C_{i j k}$ as all their components have zero speed.) Both the main and constraint system are strongly hyperbolic for $a b>0$. Note that the coefficients of $\Gamma$ in $U_{0}$ etc. are themselves left (row) eigenvectors of $P_{n}$.) The non-zero speed variables contain terms which are themselves zero speed variables, (for example, $U_{ \pm}$contains $\left.d_{q q}\right)$. is

In the base of characteristic variables the EM system

$$
\begin{aligned}
\partial_{t} d_{q q} & =\frac{1}{2} \partial^{A}\left(U_{+A}-U_{-A}\right) \\
\partial_{t} U_{0} & =-\frac{1}{2}(b-1) \partial^{A}\left(U_{+A}-U_{-A}\right) \\
\partial_{t} U_{ \pm} & = \pm \sqrt{a b} \partial_{n} U_{ \pm}-\frac{a b+c-1}{2} \partial^{A}\left(U_{+A}-U_{-A}\right)
\end{aligned}
$$




$$
\begin{aligned}
& \pm \sqrt{a b} \partial^{A}\left[\frac { c } { 2 } \left(U_{+A}\right.\right. \\
& \left.\left.+U_{-A}\right)+\left(c^{2}-1\right) d_{A n}\right] \\
\partial_{t} U_{ \pm A}= & \pm \partial_{n} U_{ \pm A}+\frac{c}{2 \sqrt{a b}} \partial_{A}\left(U_{+}-U_{-}\right) \\
& \pm \partial^{B}\left(d_{B A}-c d_{A B}\right) \\
& \mp \partial_{A}\left[\frac{a b+c-1}{2 a b}\left(U_{+}+U_{-}\right)+\frac{1-c}{b} U_{0}\right. \\
& \left.+\frac{c-1}{2 a b}(2 c-2+2 a+a b) d_{q q}\right], \\
\partial_{t} d_{A n}= & -\frac{1}{2 \sqrt{a b}} \partial_{A}\left(U_{+}-U_{-}\right), \\
\partial_{t} d_{A B}= & \frac{1}{2} \partial_{A}\left(U_{+B}-U_{-B}\right) \\
& -\frac{1}{4} q_{A B} \partial^{C}\left(U_{+C}-U_{-C}\right) .
\end{aligned}
$$

Similarly, the constraint system in characteristic variables is

$$
\begin{aligned}
\partial_{t} C_{ \pm}= & \pm \sqrt{a b} \partial_{n} C_{ \pm} \\
& \pm \sqrt{a b} \partial^{A}\left[a C_{A}+(1-c) C_{A j}{ }^{j}\right], \\
\partial_{t}\left(a C_{A}\right)= & \frac{\sqrt{a b}}{2} \partial_{A}\left(C_{+}-C_{-}\right), \\
\partial_{t} C_{i j}= & 0 \\
\partial_{t} C_{i j k}= & 0 .
\end{aligned}
$$

\section{Symmetric hyperbolicity}

The first-order EM system admits the conserved energy

$$
\epsilon=c_{0} \epsilon_{0}+c_{1} \epsilon_{1}
$$

where $c_{0}$ and $c_{1}$ are two arbitrary coefficients and

$$
\begin{aligned}
\epsilon_{0}= & E^{i} E_{i}+d^{i j} d_{i j}-c d^{i j} d_{j i}-2 a \Gamma d_{i}{ }^{i} \\
& +(c-1-a b+2 a)\left(d_{i}{ }^{i}\right)^{2}, \\
\epsilon_{1}= & {\left[\Gamma+(b-1) d_{i}{ }^{i}\right]^{2} . }
\end{aligned}
$$

The flux is

$$
F^{i}=2 c_{0}\left[a\left(\Gamma-d_{j}{ }^{j}\right) E^{i}+(1-c) d_{j}^{j} E^{i}-\left(d^{i j}-c d^{j i}\right) E_{j}\right]
$$

Note that $\epsilon_{1}$ has zero flux. $\epsilon$ and $F^{n}$ can be written in terms of characteristic variables as

$$
\begin{aligned}
\epsilon=c_{0} & {\left[d_{A B} d^{A B}-c d_{A B} d^{B A}+\left(1-c^{2}\right) d_{A n} d^{A n}\right.} \\
& +\frac{1}{2}\left(U_{+A} U_{+}^{A}+U_{-A} U_{-}^{A}\right)+\frac{1}{2 a b}\left(U_{+}^{2}+U_{-}^{2}\right) \\
& \left.+\frac{c-1}{2 a b}\left(4 a d_{q q} U_{0}+(2-4 a+a b-2 c) d_{q q}{ }^{2}\right)\right] \\
+ & \left(c_{1}-\frac{a}{b} c_{0}\right)\left[U_{0}+(b-1) d_{q q}\right]^{2}
\end{aligned}
$$

$$
\begin{aligned}
F^{n}= & \frac{1}{2} c_{0}\left[\left(U_{+A} U_{+}^{A}-U_{-A} U_{-}^{A}\right)\right. \\
& \left.+\frac{1}{\sqrt{a b}}\left(U_{+}^{2}-U_{-}^{2}\right)\right] .
\end{aligned}
$$

Now we impose that $\epsilon$ is positive definite. We can write

$$
d_{i j} d^{i j}-c d_{i j} d^{j i}=\left(\cos \varphi d_{i j}-\sin \varphi d_{j i}\right)^{2}
$$

where $\sin 2 \varphi \equiv c$. This is positive definite for $|c|<1$. Overall there are four positivity conditions:

$$
\begin{aligned}
& c_{0}>0, \quad c_{1}>\frac{3 a^{2}}{3 a b+2 c-2} c_{0}, \\
& |c|<1, \quad a b>\frac{2}{3}(1-c) .
\end{aligned}
$$

The covariant constraint energy density and flux, after fixing an overall factor, are

$$
\begin{aligned}
\epsilon_{c}= & {\left[a C_{i}+(1-c) C_{i j}{ }^{j}\right]^{2}+a b C_{E}^{2} } \\
& +w_{1}\left(C_{i j}\right)^{2}+w_{2}\left(C_{i j k}\right)^{2} \\
F_{c}^{i}= & 2 a b C_{E}\left[a C_{i}+(1-c) C_{i j}{ }^{j}\right] .
\end{aligned}
$$

It is unconditionally positive definite. In terms of characteristic variables,

$$
\begin{aligned}
\epsilon_{c}= & \frac{1}{2}\left(C_{+}^{2}+C_{-}^{2}\right)+\left[a C_{A}+(1-c) C_{A j}{ }^{j}\right]^{2} \\
& +w_{1}\left(C_{i j}\right)^{2}+w_{2}\left(C_{i j k}\right)^{2} \\
F_{c}^{n}= & \frac{1}{2} \sqrt{a b}\left(C_{+}^{2}-C_{-}^{2}\right) .
\end{aligned}
$$

The terms multiplied by $w_{1}$ and $w_{2}$ are strictly constant, and so $w_{1}>0$ and $w_{2}>0$ are arbitrary.

For the main system and the constraint system to be symmetric hyperbolic, the parameters of the system must obey the positivity conditions (110). The strong hyperbolicity condition $a b>0$, which must also be obeyed, is implied by these.

\section{Pseudo-differential reduction to first order}

\section{Field equations}

We now carry out the pseudo-differential reduction for comparison. Defining $\hat{u}$ as the Fourier transform of $u$ in space, and substituting this into the field equations we obtain

$$
\begin{aligned}
\partial_{t} \hat{A}_{i} & =-\hat{E}_{i} \\
\partial_{t} \hat{E}_{i} & =\omega_{j} \omega^{j} \hat{A}_{i}+a i \omega_{i} \hat{\Gamma}-(1-a) \omega_{i} \omega^{j} \hat{A}_{j} \\
\partial_{t} \hat{\Gamma} & =(b-1) i \omega_{i} \hat{E}^{i}
\end{aligned}
$$

The fact that the system is second order in space is indicated by the fact that the highest power of $|\omega|$ on the right-hand side is $\left|\omega^{2}\right|$. On the other hand, there is no 
power of $|\omega|$ on the right-hand side of $\partial_{t} \hat{A}_{i}$. We define new variables by absorbing powers of $|\omega|$ into some of the Fourier transforms, namely

$$
\hat{a}_{i} \equiv i|\omega| \hat{A}_{i}
$$

In these variables, the ODE system in the Fourier variables becomes.

$$
\begin{aligned}
\partial_{t} \hat{a}_{i} & =-i|\omega| \hat{E}_{i} \\
\partial_{t} \hat{E}_{i} & =i|\omega|\left[-\hat{a}_{i}+a n_{i} \hat{\Gamma}+(1-a) n_{i} n^{j} \hat{a}_{j}\right], \\
\partial_{t} \hat{\Gamma} & =i|\omega|(b-1) n_{i} \hat{E}^{i}
\end{aligned}
$$

where again $n_{i} \equiv \omega_{i} /|\omega|$. The right-hand sides are now the symbols of first-order pseudo-differential operators, but only the third is also the symbol of a differential operator.

\section{Strong hyperbolicity}

We can now find the characteristic variables of the pseudo-differential system using the same tensor decomposition as above. We write the pseudo-differential system as

$$
\partial_{t} \hat{u}=i|\omega| P_{n} \hat{u} .
$$

$P_{n}$ in (122) is a submatrix of $P_{n}$ in (78), with $E_{i} \rightarrow \hat{E}_{i}$, $\Gamma \rightarrow \hat{\Gamma}, \partial_{n} A_{i} \rightarrow i|\omega| \hat{A}_{i} \equiv \hat{a}_{i}$, but $\partial_{A} A_{i} \rightarrow 0$ because there are no transversal derivatives in the pseudo-differential framework. Therefore the rows and columns corresponding to $d_{A i}$ and $d_{q q}$ disappear. Note that with these deletions, $c$ disappears from $P_{n}$ : this must happen as $c$ parameterises an ambiguity of the differential reduction. The characteristic variables are

$$
\begin{aligned}
\hat{U}_{0} & \equiv \hat{\Gamma}+(b-1) \hat{a}_{n}, \\
\hat{U}_{ \pm} & \equiv a(\hat{\Gamma}-\hat{a}) \pm \sqrt{a b} \hat{E}_{n}, \\
\hat{U}_{ \pm B} & \equiv \hat{a}_{B} \mp \hat{E}_{B},
\end{aligned}
$$

with speeds $(0, \pm \sqrt{a b}, \pm 1)$.

The Fourier transforms of the constraints are

$$
\hat{C}_{E}=i|\omega| \hat{E}_{n}, \quad \hat{C}_{\Gamma}=\hat{\Gamma}-\hat{a}_{n} .
$$

With

$$
\hat{c}_{\Gamma} \equiv i|\omega| \hat{C}_{\Gamma}
$$

we have

$$
\begin{aligned}
\partial_{t} \hat{C}_{E} & =i|\omega| a \hat{c}_{\Gamma}, \\
\partial_{t} \hat{c}_{\Gamma} & =i|\omega| b \hat{C}_{E} .
\end{aligned}
$$

The characteristic variables are

$$
\hat{C}_{ \pm} \equiv a \hat{c}_{\Gamma} \pm \sqrt{a b} \hat{C}_{E}=i|\omega| \hat{U}_{ \pm}
$$

with speeds $\pm \sqrt{a b}$. The pseudo-differential system is strongly hyperbolic for $a b>0$.

Because of the relation of the two matrices $P_{n}$ discussed above, the characteristic variables for the pseudodifferential reduction could be obtained from those for the differential reduction by the replacements $d_{A i} \rightarrow 0$, $d_{n i} \rightarrow \hat{a}_{i}, E_{i} \rightarrow \hat{E}_{i}, \Gamma \rightarrow \hat{\Gamma}$, and $C_{E} \rightarrow \hat{C}_{E}, C_{A} \rightarrow 0$, $C_{n} \rightarrow \hat{C}_{\Gamma}, C_{i j} \rightarrow 0, C_{i j k} \rightarrow 0$.

\section{Second-order in space, first order in time}

\section{Strong hyperbolicity}

To construct the second-order characteristic variables, we consider the principal part of the evolution of $\tilde{u} \equiv$ $\left(E_{i}, \partial_{i} A_{j}, \Gamma\right)$, which obey

$$
\partial_{t} \tilde{u}=P_{n} \partial_{n} \tilde{u}+P^{A} \partial_{A} \tilde{u} .
$$

One might think that $P_{n}$ is the same matrix as in the differential first order reduction. But consider, for example, Eq. 85), which is shorthand for

$$
\partial_{t} E_{A}=-\partial_{n} d_{n A}+c \partial_{n} d_{A n}+\text { transversal derivatives }
$$

In the second-order system this becomes

$$
\partial_{t} E_{A}=-\partial_{n}^{2} A_{A}+c \partial_{n} \partial_{A} A_{n}+\text { transversal derivatives, }
$$

but we can commute derivatives and so lump $\partial_{n} \partial_{A} A_{n}=$ $\partial_{A} \partial_{n} A_{n}$ with the transversal derivatives. As $\partial_{t} \partial_{A} A_{n}=$ $\partial_{A} \partial_{t} A_{n}$ can also be considered purely transversal, we can set both the row and column corresponding to $\partial_{A} A_{n}$ in $P_{n}$ equal to zero. This is true for all $\partial_{A} A_{i}$, and $\partial A C_{\Gamma}$ in the constraint system. Therefore we are effectively diagonalising smaller matrices $P_{n}$ for the main and constraint systems. These are identical (up to variable names) to those in the pseudo-differential reduction.

The characteristic variables for the second-order EM system obtained by diagonalising this reduced $P_{n}$ are

$$
\begin{aligned}
U_{0} & \equiv \Gamma+(b-1) \partial_{n} A_{n} \\
U_{ \pm}^{\prime} & \equiv a\left(\Gamma-\partial_{n} A_{n}\right) \pm \sqrt{a b} E_{n} \\
U_{ \pm B}^{\prime} & \equiv \partial_{n} A_{B} \mp E_{B}
\end{aligned}
$$

with speeds $(0, \pm \sqrt{a b}, \pm 1)$ for the main system, and

$$
C_{ \pm} \equiv a \partial_{n} C_{\Gamma} \pm \sqrt{a b} C_{E}
$$

with speeds $\pm \sqrt{a b}$ for the constraint system. To complete the set of characteristic variables, we add the transversal derivatives

$$
\partial_{A} A_{i}, \quad \partial_{A} C_{\Gamma},
$$

which are by definition zero-speed variables. 


\section{Symmetric hyperbolicity}

We obtain a conserved energy for the second-order system by writing down the most general scalar $\epsilon$ that is quadratic in $\Gamma, E_{i}$ and $\partial_{i} A_{j}$ and adjusting the free coefficients to obtain conservation. The result is

$$
\begin{aligned}
\epsilon= & c_{0} \epsilon_{0}+c_{1} \epsilon_{1}+c_{2} \epsilon_{2}, \\
\epsilon_{0}= & E_{i}^{2}+\left(\partial_{i} A_{j}\right)^{2}-2 a \Gamma \partial^{i} A_{i} \\
& +(2 a-1-a b)\left(\partial^{i} A_{i}\right)^{2}, \\
\epsilon_{1}= & {\left[\Gamma+(b-1) \partial^{i} A_{i}\right]^{2}, } \\
\epsilon_{2}= & \left(\partial^{i} A_{i}\right)^{2}-\partial_{i} A_{j} \partial^{j} A^{i} .
\end{aligned}
$$

The flux is given by

$$
\begin{aligned}
& F_{0}^{i}=2\left\{\left[\left(a \gamma+(1-a) \partial^{j} A_{j}\right] E^{i}-\partial^{i} A^{j} E_{j}\right\}\right. \\
& F_{2}^{i}=2\left(\partial^{i} A^{j} E_{j}-\partial^{j} A_{j} E^{i}\right)
\end{aligned}
$$

while $\epsilon_{1}$ has no flux. The constraint energy is unique up to an overall factor, and is given by

$$
\begin{aligned}
\epsilon_{c} & =a^{2} \partial_{i} C_{\Gamma} \partial^{i} C_{\Gamma}+a b C_{E}^{2}, \\
F_{c}^{n} & =2 a^{2} b C_{\Gamma} \partial^{i} C_{E} .
\end{aligned}
$$

If we add zero-speed terms $\partial_{A} A_{i}$ to $U_{ \pm}^{\prime}$ and $U_{ \pm A}^{\prime}$ as follows,

$$
\begin{aligned}
U_{ \pm} \equiv & a\left(\Gamma-\partial_{n} A_{n}-\partial^{B} A_{B}\right) \pm \sqrt{a b} E_{n} \\
& +(1-c) \partial^{B} A_{B}, \\
U_{ \pm B} \equiv & \partial_{n} A_{B} \mp E_{B}-c \partial_{B} A_{n},
\end{aligned}
$$

where $c \equiv c_{2} / c_{0}$, we can write the main flux in the form (108), and with (137) we can write the constraint flux in the form (114). Furthermore, the evolution equations expressed in the second-order characteristic variables are given by (93 98), with the obvious replacements. This works because the same constant $c$ arises twice in different ways: in the first-order system it parameterises an arbitrariness of writing the second-order field equations in terms of first-order variables, and this is passed on to the energy. In the second-order system there is no such ambiguity in the field equations, but $c$ arises as the free coefficient $c_{2} / c_{0}$ in the energy.

\section{E. Constraint-preserving boundary conditions}

The following discussion applies equally to the firstorder and second-order symmetric hyperbolic systems. The constraint-preserving boundary conditions we are about to derive contain only time derivatives when applied to the first-order system, but both space and time derivatives when applied to the second-order system. We shall explicitly write down expressions for the first-order system. The corresponding expressions for the second order system are obtained by the replacement $d_{i j} \rightarrow \partial_{i} A_{j}$, and interpreting the characteristic variables as those of the second-order system.
The maximally dissipative boundary conditions on the main variables are

$$
\begin{aligned}
U_{+}-\kappa_{1} U_{-} & =f, \\
U_{+A}-\kappa_{2} U_{-A} & =f_{A},
\end{aligned}
$$

where $\left|\kappa_{1}\right| \leq 1$ and $\left|\kappa_{2}\right| \leq 1$, and $f$ and $f_{A}$ are given functions. We are only interested in solutions with vanishing constraints. Therefore we formally impose homogeneous maximally dissipative boundary conditions on the constraint system, that is

$$
C_{+}-\kappa_{3} C_{-}=0
$$

with $\left|\kappa_{3}\right| \leq 1$. We now show how these boundary conditions can be made consistent, following the general procedure set out in Sec. $\mathbf{\nabla}$

$C_{ \pm}$can be expressed in terms of the main characteristic variables as

$$
\begin{aligned}
C_{ \pm}= & \partial_{n} U_{ \pm}+\frac{1}{2} \partial^{A}\left[\mp \sqrt{a b}\left(U_{+A}-U_{-A}\right)\right. \\
& \left.+(c-1)\left(U_{+A}+U_{-A}+2 c d_{A n}\right)\right] .
\end{aligned}
$$

We use the evolution equation for $U_{ \pm}$to replace $\partial_{n} U_{ \pm}$ by $\partial_{t} U_{ \pm}$. Eq. (151) then becomes

$$
\begin{aligned}
& \partial_{t}\left(U_{+}+\kappa_{3} U_{-}\right) \\
= & \frac{\left(1+\kappa_{3}\right)(1-c)}{2} \partial^{A}\left(U_{+A}-U_{-A}\right) \\
& +\frac{\left(1-\kappa_{3}\right) \sqrt{a b}}{2} \partial^{A}\left[U_{+A}+U_{-A}+2(c-1) d_{A n}\right] .
\end{aligned}
$$

We impose this as an evolution equation for an auxiliary field $X \equiv U_{+}+\kappa_{3} U_{-}$on the boundary. We then impose the boundary condition (149) on $U_{+}$with $\kappa_{1}=-\kappa_{3}$ and $f=X$. We also impose the maximally dissipative boundary conditions (150) on $U_{+A}$, which represent the two polarisations of ingoing radiation. $\kappa_{2}$ and $f_{A}$ must therefore be chosen on physical grounds: stability only dictates $\left|\kappa_{2}\right| \leq 1$.

\section{Dirichlet boundary conditions}

As discussed in general in Sec. $\mathbf{D}$ one can demonstrate well-posedness with these constraint-preserving boundary conditions if the bulk system does not couple back to the main system. There are two cases in which this can be achieved: For $\kappa_{3}=1, d_{A n}$ is eliminated from the boundary system, while for $\kappa_{3}=-1$, its evolution equation(97) becomes part of an expanded closed boundary system. For $\kappa_{3}=\kappa_{2}=-\kappa_{1}=1$, the boundary system and boundary conditions are

$$
\begin{aligned}
\partial_{t} X & =(1-c) \partial^{A} f_{A}, \\
U_{+}+U_{+} & =X, \\
U_{+A}-U_{-A} & =f_{A},
\end{aligned}
$$

where $f_{A}$ is free data. The boundary condition on $U_{ \pm}$ corresponds to Dirichlet boundary conditions on $C_{E}$. 


\section{Neumann boundary conditions}

For $\kappa_{3}=\kappa_{2}=-\kappa_{1}=-1$, the boundary system and the boundary conditions for the main system are

$$
\begin{aligned}
\partial_{t} X & =\sqrt{a b} \partial^{A}\left[f_{A}+2(c-1) d_{A n}\right], \\
\partial_{t} d_{A n} & =-\frac{1}{2 \sqrt{a b}} \partial_{A} X, \\
U_{+}-U_{-} & =X \\
U_{+A}+U_{-A} & =f_{A} .
\end{aligned}
$$

The boundary condition on $U_{ \pm}$corresponds to Neumann boundary conditions on $C_{\Gamma}$.

The boundary system is symmetric hyperbolic with the energy density, flux, and source term

$$
\begin{aligned}
\epsilon_{b} & =X^{2}+4 a b(1-c)\left(d_{A n}\right)^{2}, \\
\partial_{t} \epsilon_{b} & =\partial_{A} F_{b}^{A}+s_{b}, \\
F_{b}^{A} & =4 \sqrt{a b}(c-1) X d_{A n}, \\
s_{b} & =2 \sqrt{a b} X \partial^{A} f_{A} .
\end{aligned}
$$

The characteristic variables of the boundary system in the direction $m_{A}$ are

$$
X_{ \pm} \equiv X \mp 2 \sqrt{a b(1-c)} d_{m n}
$$

with speeds $\pm \sqrt{1-c}$. The boundary energy is controlled by

$$
\begin{aligned}
\frac{d E_{b}}{d t} & =\int_{\partial \Omega} s d S+\int_{\partial \partial \Omega} F_{b}^{m} d s, \\
F_{b}^{m} & =\frac{1}{2} \sqrt{1-c}\left(X_{+}^{2}-X_{-}^{2}\right),
\end{aligned}
$$

where $m_{A}$ is the normal to $\partial \partial \Omega$ in $\partial \Omega$.

For a smooth boundary $(\partial \partial \Omega=0)$, the boundary energy can therefore be estimated by

$$
\frac{d E_{b}}{d t} \leq 2 \sqrt{a b}\|X\|\left\|\partial^{A} f_{A}\right\| \leq 2 \sqrt{a b} \sqrt{E_{b}}\left\|\partial^{A} f_{A}\right\|
$$

For a cubic boundary, one also has to consider the $\partial \partial \Omega$ terms at the edges of each face. From (160) and (90) we have

$$
d_{m n}=\frac{f_{n}^{(m)}+c f_{m}^{(n)}}{1-c^{2}}
$$

where $f_{A}^{(n)}$ are the free data on the boundary with normal $n_{i}$. They are already given. This leaves us no choice but to impose Dirichlet boundary conditions given by (169) on $d_{m n}$ at the edge with normal $m_{A}$ of the face with normal $n_{i}$. From (165) and (167) we see that these are maximally dissipative boundary conditions on the system on each face, so that the estimate (168) still holds. Therefore the coupled bulk/boundary system is well-posed in the Neumann case.

\section{Laplace-Fourier analysis}

With our current methods we can decouple the boundary system from the bulk system, and hence prove wellposedness of the initial-boundary value problem, only for the two extreme values $\kappa_{3}= \pm 1$. The same was found previously in imposing constraint-preserving boundary conditions on the linearised Einstein-Christoffel system 7] and on the full Einstein equations in harmonic gauge [9]. But the lack of a proof does not rule out that for example the Sommerfeld boundary conditions with $\kappa_{3}=0$ could also be well-posed.

To investigate this possibility further, we now use the Laplace-Fourier method to explicitly check for modes that grow arbitrarily rapidly. The EM equations are untypical in that they are linear with constant coefficients. For a quasilinear system such as the Einstein equations, we use the high frequency, or frozen coefficients, approximation, and consider only the principal part of the evolution equations and constraints, thus recovering a linear system with constant coefficients. In the frozen coefficients approximation we assume that the linearised perturbation varies over space and time scales much smaller than those given by the background solution and the numerical domain. For consistency we must therefore assume that the domain is a half space and that the boundary is a plane. Without loss of generality, we assume that the evolution domain is $-\infty<x^{1} \leq 0$ and $-\infty<x^{A} \equiv\left(x^{2}, x^{3}\right)<\infty$. The general solution with homogeneous boundary conditions can then be written as a sum of modes of the form

$$
u\left(x^{i}, t\right)=e^{s t+i \omega_{A} x^{A}+\lambda x^{1}} v
$$

where $v$ is a constant eigenvector to be determined. (If the corresponding eigenvalue $\lambda$ was degenerate, $v$ would be a polynomial in $x^{1}$ rather than constant, but this does not happen in for the EM system.) If the boundary conditions are inhomogeneous, we must add to this ansatz a particular integral that does not concern us here because its growth is controlled by the boundary data. We are interested only in modes that are square-integrable over space at any moment in time. Therefore we assume that $\omega_{A}$ is real, and that $\operatorname{Re} \lambda \geq 0 . s$ and $\lambda$ will in general be complex.

If a mode of this form exists for some $\left(s, \omega_{A}, \lambda, v\right)$, then one exists also for $\left(k s, k \omega_{A}, k \lambda, v\right)$ for any $k>0$. Therefore, if any growing mode exists, there are growing modes with arbitrarily large growth rates $k s$, and the problem is ill-posed. A necessary condition for well-posedness of the initial-boundary value problem is therefore that the homogeneous boundary conditions rule out the existence of any mode with Res $>0$ for all real $\omega_{A}$ and all $\lambda$ with $\operatorname{Re} \lambda \geq 0$.

We now examine our proposed constraint-preserving boundary conditions for the EM system. To simplify the algebra, we restrict ourselves to the case $a=b=1$, $c=0$ (which is symmetric hyperbolic). The evolution 
equations for the characteristic variables simplify to

$$
\begin{aligned}
\partial_{t} d_{q q} & =\frac{1}{2} \partial^{A}\left(U_{+A}-U_{-A}\right), \\
\partial_{t} U_{0} & =0 \\
\partial_{t} d_{A n} & =-\frac{1}{2} \partial_{A}\left(U_{+}-U_{-}\right), \\
\partial_{t} d_{A B} & =\frac{1}{2} \partial_{A}\left(U_{+B}-U_{-B}\right)-\frac{1}{4} q_{A B} \partial^{C}\left(U_{+C}-U(17)^{4}\right) \\
\partial_{t} U_{ \pm} & = \pm \partial_{n} U_{ \pm} \mp \partial^{A} d_{A n}, \\
\partial_{t} U_{ \pm A} & = \pm \partial_{n} U_{ \pm A} \pm \partial^{B} d_{B A} \mp \partial_{A} U_{0} \pm \frac{1}{2} \partial^{A} d_{q q}
\end{aligned}
$$

(Once again, we could trivially rewrite this for the second-order system). The constraint-preserving boundary conditions are

$$
\begin{aligned}
\partial_{t}\left(U_{+}+\kappa_{3} U_{-}\right)= & \partial^{A}\left[U_{+A}-\kappa_{3} U_{-A}\right. \\
& \left.-\left(1-\kappa_{3}\right) d_{A n}\right], \\
U_{+A}-\kappa_{2} U_{-A}= & 0 .
\end{aligned}
$$

Using the Laplace-Fourier ansatz in the evolution equations, we obtain a linear eigenvalue problem for $v$ with eigenvalue $\lambda(s,|\omega|)$. Its solution is

$$
\begin{aligned}
\left(\begin{array}{c}
U_{+} \\
U_{-}
\end{array}\right) & =\gamma_{1} e^{s t+i \omega_{A} x^{A}+\lambda x^{1}}\left(\begin{array}{c}
1 \\
\psi^{2}
\end{array}\right), \\
\left(\begin{array}{c}
U_{A+} \\
U_{A-}
\end{array}\right) & =\gamma_{2 A} e^{s t+i \omega_{A} x^{A}+\lambda x^{1}}\left(\begin{array}{c}
1 \\
\psi^{2}
\end{array}\right),
\end{aligned}
$$

where $\lambda=\sqrt{s^{2}+|\omega|^{2}}$ and $\psi=\sqrt{z^{2}+1}-z$ with $z \equiv$ $s /|\omega| . \quad \gamma_{1}$ and $\gamma_{2 A}$ are free coefficients. We ensure that $\operatorname{Re} \lambda>0$ for $\operatorname{Re} s>0$ by putting the branch cut for the square root on the negative real axis.

Applying the boundary condition (178) to (180) gives the algebraic condition $\gamma_{2 A}\left(\kappa_{2} \psi^{2}-1\right)=0$. This means that $\gamma_{2 A}=0$ unless $\kappa_{2} \psi^{2}(z)=1$. Now $\psi^{2}(z)$ maps the right half-plane into the unit disk with the negative real axis removed. Therefore, $\kappa_{2} \psi^{2}(z)=1$ for $\operatorname{Re} z>0$ is ruled out if $\left|\kappa_{2}\right| \leq 1$. The boundary condition (177) applied to (180) gives a more complicated algebraic condition linking $\gamma_{1}$ and $\omega^{A} \gamma_{2 A}$. However, with $\gamma_{2 A}=0$ this simplifies, after some algebra, to $\gamma_{1}\left(\kappa_{3} \psi^{2}-1\right)=0$, and so we must also impose $\left|\kappa_{3}\right| \leq 1$.

Our calculation has shown that the initial-boundary value problem has no growing square-integrable modes of the form (179180) for $\left|\kappa_{2}\right| \leq 1$ and $\left|\kappa_{3}\right| \leq 1$. This is consistent with our energy method proof that the EM initial-boundary value problem is well-posed for $\kappa_{3}= \pm 1$ and $\left|\kappa_{2}\right| \leq 1$, but it also suggests that the problem is well-posed in the more general case, and we expect that a proof of this can be found using a modified energy.

\section{CONCLUSIONS}

We have proposed a new method for setting up a wellposed initial-boundary value problem for evolution sys- tems, such as variants of the ADM equations, that are first order in time but second order in space. One might call it the second-order energy method. It involves two steps:

1. Find a complete set of characteristic variables for any given direction that obey Eq. (34). They are not yet unique. This is our definition of strong hyperbolicity.

2. Find a conserved covariant energy, and express it and the corresponding flux in terms of the characteristic variables. This can be done for a unique set of characteristic variables. This is our definition of symmetric hyperbolicity.

Our definition of strong hyperbolicity for a secondorder system is equivalent to that using a pseudodifferential reduction to first order [1, 2], as both definitions require the same matrix to be diagonalisable. But the pseudo-differential method cannot be extended to symmetric hyperbolicity, roughly speaking because Fourier transforms do not allow for boundaries in space. Our definition of symmetric hyperbolicity for a secondorder system is similar to that for the differential reduction to first order, but it does not introduce auxiliary variables and constraints, and so does not enlarge the solution space.

We have worked through both types of first-order reduction, as well as the second-order energy method, in the example of electromagnetism, in order to illustrate the rather subtle similarities and differences. In a companion paper [10] we shall use the energy method to prove symmetric hyperbolicity and suggest constraintpreserving boundary conditions for variants of the ADM equations.

\section{Acknowledgments}

The authors would like thank G Nagy, O Ortiz and O Reula for communicating a draft paper and for discussions. CG would like to thank the Kavli Institute for Theoretical Physics for hospitality while this work was begun, and T Baumgarte, G Calabrese, H Friedrich, L Kidder, L Lindblom, O Sarbach, M Scheel, D Shoemaker and J Vickers for helpful discussions. JMM was supported by the Comunidad Autónoma de Madrid and Fondo Social Europeo and also in part by the Spanish MCYT under the research project BFM2002-04031-C0202.

\section{APPENDIX A: AN ALTERNATIVE FIRST-ORDER REDUCTION}

Lindblom et al 11] have imposed constraint-preserving boundary conditions of the Sommerfeld type on a symmetric hyperbolic first-order reduction of the EM system (without proving well-posedness of the resulting initialboundary value problem). They consider the following 
first-order reduction of the EM system:

$$
\begin{aligned}
& \partial_{t} E_{i}=\partial^{j}\left(d_{i j}-d_{j i}\right)+\frac{\gamma_{1}}{2}\left(\partial_{i} d_{j}{ }^{j}-\partial^{j} d_{i j}\right), \\
& \partial_{t} d_{i j}=-\partial_{i} E_{j}+\gamma_{2} \delta_{i j} \partial^{k} E_{k} .
\end{aligned}
$$

This corresponds to our first-order reduction with $a=$ 0 (and $\Gamma$ therefore not part of the system) and $c=$ $1-\left(\gamma_{1} / 2\right)$, plus the additional term parameterised by $\gamma_{2}$. The latter plays a similar role to our parameter $b$, in adding the constraint $C_{E}$ to the evolution equation for $d_{i}{ }^{i}(=\Gamma)$. They find that the system is strongly hyperbolic for $\gamma_{1} \gamma_{2}>0$, and symmetric hyperbolic for $0<\gamma_{1}<4$ and $\gamma_{2}>1 / 3$. However, this first-order reduction has as its second-order counterpart only the original EM system (6061), which is only weakly hyperbolic. The term multiplied by $\gamma_{1}$ vanishes identically because partial derivatives can be commuted, and the term $\gamma_{2}$ cannot arise if we evolve $A_{i}$ rather than $d_{i j}$. In hindsight, one can see that both terms have second-order counterparts as long as we have the trace of $d_{i j}$ as an auxiliary variable: the rest of $d_{i j}$ is not required.
[1] $\mathrm{H}$ Kreiss and OE Ortiz, in The conformal structure of spacetimes: Geometry, Analysis, Numerics, eds J Frauendiener and H Friedrich, Springer (Lecture Notes in Physics vol. 604), Heidelberg, 2002.

[2] G Nagy, OE Ortiz and OA Reula, Strongly hyperbolic second order Einstein's evolution equation, in preparation.

[3] B Gustafsson, H-O Kreiss and J Oliger, Time-dependent problems and difference methods, John Wiley, New York 1995.

[4] J. M. Stewart, Class. Quantum Grav. 15, 2865 (1998).

[5] O Reula, Living Reviews in Relativity, 1998-3.

[6] G Calabrese and O Sarbach, J. Math. Phys. 44, 3888
(2003).

[7] G Calabrese et al, Commun. Math. Phys. 240, 377 (2003).

[8] AM Knapp, EJ Walker and TW Baumgarte, Phys. Rev. D 65, 064031 (2002).

[9] B Szilagyi and J Winicour, Phys. Rev. D 68, 041501 (2003).

[10] C Gundlach and J M Martín-García, Symmetric hyperbolic second-order Einstein equations, submitted to Phys. Rev. D.

[11] L Lindblom et al, Controlling the growth of constraints in hyperbolic evolution systems, e-print gr-qc/0402027 